\title{
Usefulness of combined endobronchial and endoscopic ultrasound-guided needle aspiration in the diagnosis of sarcoidosis: a prospective multicenter trial
}

\author{
Anna Filarecka ${ }^{1}$, Maciej Gnass ${ }^{1}$, Jacek Wojtacha², Jerzy Soja ${ }^{3}$, Juliusz Pankowski ${ }^{4}$, \\ Damian Czyżewski² ${ }^{2}$, Wojciech Zajęcki ${ }^{5}$, Arkadiusz Joks ${ }^{6}$, Adam Ćmiel , Artur Szlubowski1 ${ }^{1,2,6}$ \\ 1 Endoscopy Unit, Pulmonary Hospital, Zakopane, Poland \\ 2 Chair and Department of Thoracic Surgery of Silesian Medical University, Katowice, Poland \\ Department of Medicine, Jagiellonian University, Kraków, Poland \\ Department of Pathology, Pulmonary Hospital, Zakopane, Poland \\ Department of Pathology, Silesian University, Katowice, Poland \\ 6 Izerian Center of Pulmonology and Chemotherapy, Szklarska Poręba, Poland \\ 7 Department of Applied Mathematics, AGH University of Science and Technology, Kraków, Poland
}

\section{KEY WORDS}

bronchoscopy,

cell block,

endosonography,

sarcoidosis

\section{EDITORIAL}

by Trisolini, see p. 568
Correspondence to:

Maciej Gnass, MD, PhD,

Endoscopy Unit, Pulmonary Hospital, ul. Gładkie 1, 34-500 Zakopane,

Poland, phone: +48182015045,

e-mail:m.gnass@gmail.com

Received: April 25, 2020.

Revision accepted: May 15, 2020

Published online: May 28, 2020.

Pol Arch Intern Med. 2020;

130 (7-8): 582-588

doi:10.20452/pamw.15399

Copyright by the Author(s), 2020

\section{ABSTRACT}

INTRODUCTION Needle biopsy of enlarged lymph nodes is an accepted method for the diagnostic workup of sarcoidosis, but the optimal endosonography-guided approach is yet to be determined.

OBJECTIVES The aim of our study was to assess the relative diagnostic yield of combined ultrasound-guided needle aspiration (CUS-b-NA), which includes endobronchial ultrasound-guided transbronchial needle aspiration (EBUS-TBNA) with endoscopic ultrasound fine-needle aspiration (EUS-b-FNA), as well as the role of the cell block (CB) technique and lymph node localization in the diagnostic workup of sarcoidosis. PATIENTS AND METHODS This was a prospective multicenter study including consecutive patients with clinical suspicion of stage I or II sarcoidosis. CUS-b-NA with smears and CB technique were performed in the whole study group. If a biopsy result was not conclusive, an invasive diagnostic workup and a 6-month follow-up were scheduled.

RESULTS Out of 77 screened patients, 54 signed written consent and 50 were enrolled for the final analysis. The overall sensitivity of EBUS-TBNA, EUS-b-FNA, and CUS-b-NA was $76.6 \%, 70.2 \%$, and $91.7 \%$, respectively. There were no differences between EBUS-TBNA and EUS-b-FNA $(P=0.52)$ but CUS-b-NA had a higher diagnostic yield $(P=0.005$ and $P=0.001$, respectively). Adding the $\mathrm{CB}$ method to smear technique $(P=0.008)$ and biopsy of the subcarinal lymph nodes increased the diagnostic yield $(P=0.001)$.

CONCLUSIONS The diagnostic yield of CUS-b-NA is higher than that of endosonographic techniques alone in the diagnostic workup of stage I and II sarcoidosis. The preparation of cytological material including $\mathrm{CB}$ and the choice of the subcarinal lymph node station for the biopsy increase the diagnostic efficacy.

INTRODUCTION Sarcoidosis is a chronic inflammatory disease of unknown etiology. Noncaseating granulomas in various organs, with predilection for mediastinal and hilar lymph nodes and lungs, are characteristic of this disease. ${ }^{1,2}$ The diagnosis of sarcoidosis is based on a combination of clinical outcome, radiology findings, and presence of noncaseating granulomas in involved organs confirmed by a cyto- or histopathological examination. ${ }^{2,3,4}$ Because granulomatous inflammation can be observed in many other diseases with similar clinical and / or radiological features (ie, tuberculosis, mycobacterial or fungal infections, pneumoconiosis, hypersensitivity pneumonia, lymphoma, lung cancer, berylliosis, and also sarcoid reaction, which may accompany or precede 


\section{WHAT'S NEW?}

To the best of our knowledge, this is the first study in patients with lymphadenopathy suspected of sarcoidosis to investigate the diagnostic yield of the combined approach with endobronchial ultrasound and endoscopic ultrasound with the use of a single endobronchial ultrasound scope at the same session. Commonly, transesophageal and transbronchial approaches were used as a single alternative procedure. Moreover, data about the efficacy of endoscopic ultrasound with the use of ultrasound bronchoscope in sarcoidosis are still poor. Our data showed that biopsy of the subcarinal lymph nodes improved the diagnostic yield. Moreover, the analysis of the techniques of processing specimens (smears and cell blocks) demonstrated that their efficacy is comparable but when used together, it was greater. Our results might be considered relevant in further analyses and helpful in designing studies on minimally invasive diagnostic workup of patients with lymphadenopathy suspected of sarcoidosis.

neoplastic disease), the exclusion of these conditions is mandatory for the final diagnosis. In order to avoid false-positive results of cytological biopsy, at least 6-month follow-up after the histological assessment is also necessary. ${ }^{4,5}$

During the last decade, the standard procedure in the diagnostic workup of stage I and II sarcoidosis became fine-needle aspiration (FNA) of the enlarged mediastinal lymph nodes (transbronchial needle aspiration [TBNA] / FNA) under control of endobronchial ultrasound (EBUS) and endoesophageal ultrasound (endoscopic ultrasound with the use of ultrasound bronchoscope [EUS-b]). ${ }^{6}$ The advantage of these methods over the conventional bronchoscopic techniques was confirmed in a few studies. ${ }^{7-10}$

Some studies showed an increase in the diagnostic yield while EBUS-TBNA and EUS-b-FNA were performed in the same patient. ${ }^{7,10}$ However, the second technique was used in the second session in case of failure of the first one. A study with a small group (33 patients) showed usefulness and safety of EUS-b-FNA in the diagnostic workup of stage I and II sarcoidosis. ${ }^{11}$

Combined ultrasound needle aspiration (CUS-b-NA) also defined as complete endosonography is the recommended technique in minimally invasive staging of patients with lung cancer. ${ }^{12}$ However, there are no data about the usefulness of this method in investigating benign lymphadenopathy (including sarcoidosis).

Biopsies guided by EBUS and EUS-b are performed with cytological 21- or 22-gauge needles and obtained specimens are used to prepare cytological smears. Flex 19-gauge needles or histological 25-gauge pro-core needles can be used to obtain more tissue material, but a positive influence on the diagnostic yield was not shown..$^{13,14}$

Diagnostic yields presented in the literature are between $71 \%$ and $94 \%$ for EBUS-TBNA ${ }^{1,4,8}$ and between $73 \%$ and $96 \%$ for EUS-b-FNA..$^{11,13}$

Although EBUS-TBNA allows biopsy of a majority of mediastinal (2R, 2L, 4R, 4L, 7) and hilar $(10 \mathrm{R}, 10 \mathrm{~L}, 11 \mathrm{R}, 11 \mathrm{~L})$ lymph node stations in the diagnostic workup of sarcoidosis, most frequently subcarinal (station 7) lymph nodes are biopsied. There are no data with regards to the preferable lymph node stations to obtain the most diagnostic material in biopsy.

There are also few studies validating the diagnostic yield of a cytological technique called cell block (CB) for the diagnostic workup of sarcoidosis, in which material obtained with needle biopsy, after fixation, is embedded in paraffin wax and sectioned for microscopic evaluation. 4,7

The aims of the study were: 1 ) to evaluate the diagnostic yield of CUS-b-NA in comparison with EBUS-TBNA and EUS-b-FNA in identification of granulomas in the enlarged intrathoracic lymph nodes; 2) to evaluate the value of the $C B$ technique in the diagnostic workup of sarcoidosis and its comparison with a standard smear technique; 3 ) to establish whether there is an association between the lymph node station and diagnostic yield of the biopsy.

PATIENTS AND METHODS This was a prospective multicenter study carried out in 3 pulmonary hospitals in Poland: Pulmonary Hospital in Zakopane, Department of Thoracic Surgery of Silesian Medical University in Katowice, and Izerian Center of Pulmonology and Chemotherapy in Szklarska Poręba. The study design was approved by the bioethical committee (no. 129/KBL/OIL/2017). All patients received detailed information regarding the study. The procedures, including the expected results and potential risks, were discussed with patients, and their informed consent was obtained.

The inclusion criteria were: age over 18 years, clinical suspicion of stage I or II sarcoidosis, and signed written informed consent. The exclusion criteria were: lack of patient consent to undergo endoscopic bioptic techniques or mediastinoscopy (MS), contraindications to bronchoscopy, coagulation disorders, and pregnancy.

CUS-b-NA procedures were performed by $4 \mathrm{ex}-$ perienced endoscopists, mainly pulmonologists, with the assistance of 2 endoscopy nurses. $\mathrm{Pa}$ tients were in the supine position, and conscious sedation with midazolam $(2.5-7.5 \mathrm{mg})$ and fentanyl (0.05-0.1 mg) administered intravenously was used. A pulse oximetry and electrocardiography monitoring was mandatory and oxygen supply was provided when necessary. Flexible videobronchoscopes with an integrated convex ultrasound probe (BF-UC180F Olympus Medical Systems Corporation, Tokyo, Japan) enabling the assessment of mediastinal and hilar structures were used for all procedures. Both FNAs of the lymph nodes, EBUS-TBNA and EUS-b-FNA, were performed in 1 session in each patient. A cytological 22-gauge 40-mm needle (NA-201SX-4022, Olympus Medical Systems Corporation, Tokyo, Japan) was used for biopsies.

In each patient, 3 to 5 biopsies of at least 2 lymph node stations were taken transbronchially as the first procedure and then also 3 to 5 biopsies 

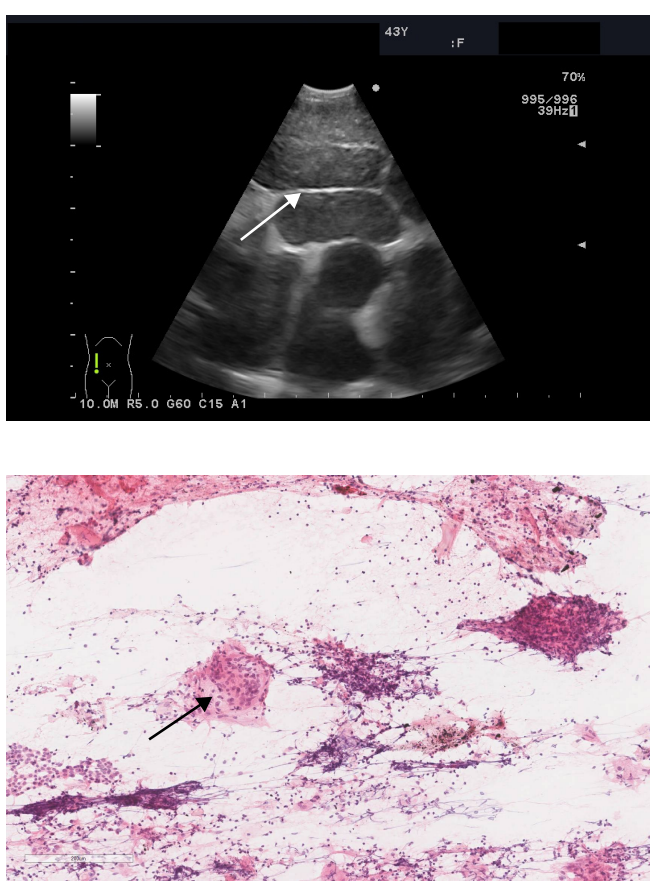

FIGURE 2 Cytological smear of a lymph node sample from a patient with sarcoidosis showing groups of epithelioid histiocytes forming a granuloma (standard hematoxylin and eosin staining; permission and image courtesy of Department of Pathology, Pulmonary Hospital, Zakopane, Poland)

of at least 2 lymph node stations were taken transesophageally at the same session. EUS-b-FNA of the subcarinal lymph node is presented in FIGURE 1.

The obtained tissue material was used to prepare smears (at least 2 for each lymph node station) and CBs (one for each biopsy method from all biopsied lymph node stations) for cytology and tuberculosis culture. Standard hematoxylin and eosin staining was used for cytological smears. Specimens for CBs were fixed with cytospin collection fluid and then processed as histological material. The analysis of cytological material was performed by 2 independent pathologists. A specimen with the lymph node tissue or / and presence of the granulomas was considered diagnostic. A cytological presentation of the sarcoid granulomas is presented in FIGURE 2 (smear) and FIgURE 3 (CB). If it was not possible to establish a diagnosis due to lack or low number of cells, the specimen was considered nondiagnostic.

In case of a biopsy result negative for granulomas, patients with a clinical diagnosis of stage I sarcoidosis were scheduled for MS and those with stage II for bronchoscopy with endobronchial mucous biopsy (EBB) and transbronchial lung biopsy (TBLB), and if still negative, for transbronchial lung cryobiopsy (TBLC). ${ }^{15}$ All enrolled patients were followed for at least 6 months after biopsy. The study protocol was presented in FIGURE 4.

Statistical analysis The sensitivity, specificity, accuracy, positive predictive value, and negative predictive value (including 95\% exact Cloper-Pearson
FIGURE 1 Endoscopic ultrasound (EUS-b) of the lymph node station 7 showing the typical pattern of lymph nodes with marked capsules (arrow) (permission and image courtesy of Endoscopy Unit, Pulmonary Hospital, Zakopane, Poland)

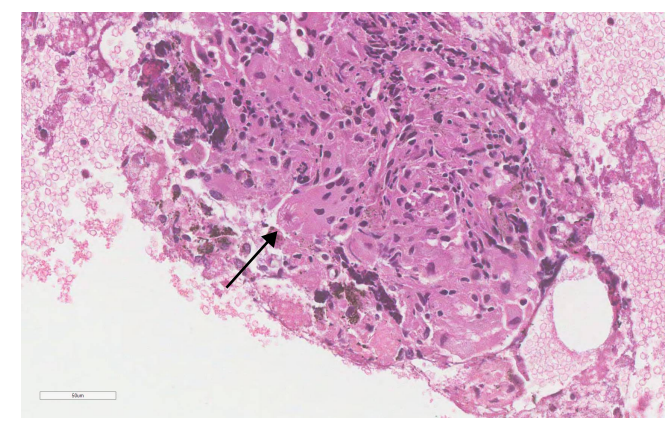

FIGURE 3 Cell block section of a lymph node sample from a patient with sarcoidosis showing a fragment of granuloma formed of epithelioid histiocytes with an asteroid body (arrow) (permission and image courtesy of Department of Pathology, Pulmonary Hospital,

Zakopane, Poland)

confidence interval [CI]) were calculated using the MEDCALC statistical software (MedCalc Software Ltd., Ostend, Belgium). The MEDCALC software and McNemar exact test were also used to compare paired proportions. The bootstrap test in the Statistica v13 environment (StatSoft Polska Sp. z o.o., Kraków, Poland) was used to compare sensitivity and accuracy of the diagnostic tests. The significance level was set at a $P$ value of less than 0.05 .

RESULTS From November 2017 to September 2019, 77 consecutive patients suspected of sarcoidosis were recruited to the study but 23 of them refused to undergo the combined procedure and bronchoscopy with TBLB / TBLC or MS as confirmatory tests. Four patients suspected of stage I sarcoidosis were excluded from further assessment. In 3 patients with spontaneous remission of adenopathy suggesting inflammation, the obtained cytological material was inadequate to prepare CBs. One patient did not agree to undergo combined procedure but only EBUS-TBNA during the examination. The final analysis was performed in 50 patients (33 men and 17 women at a mean [SD] age of 42 [8.5] years) with the clinical diagnosis of stage I sarcoidosis, 25 (50\%), and stage II sarcoidosis, 25 (50\%). A total of 12 patients (24\%) in stage I and 3 patients (6\%) in stage II had typical symptoms of Löfgren syndrome. A total of 17 patients (34\%) with stage II of the disease presented a predominance of diffuse pulmonary 
FIGURE 4 Flowchart of patients enrolled in the study

Abbreviations: CUS-b-NA, combined ultrasound-guided needle aspiration; $E B B$, endobronchial biopsy; EBUS-TBNA, ultrasound-guided transbronchial needle aspiration; EUS-b-FNA, endoscopic ultrasound fine-needle aspiration; TBLB, transbronchial lung biopsy; TBLC, transbronchial lung cryobiopsy

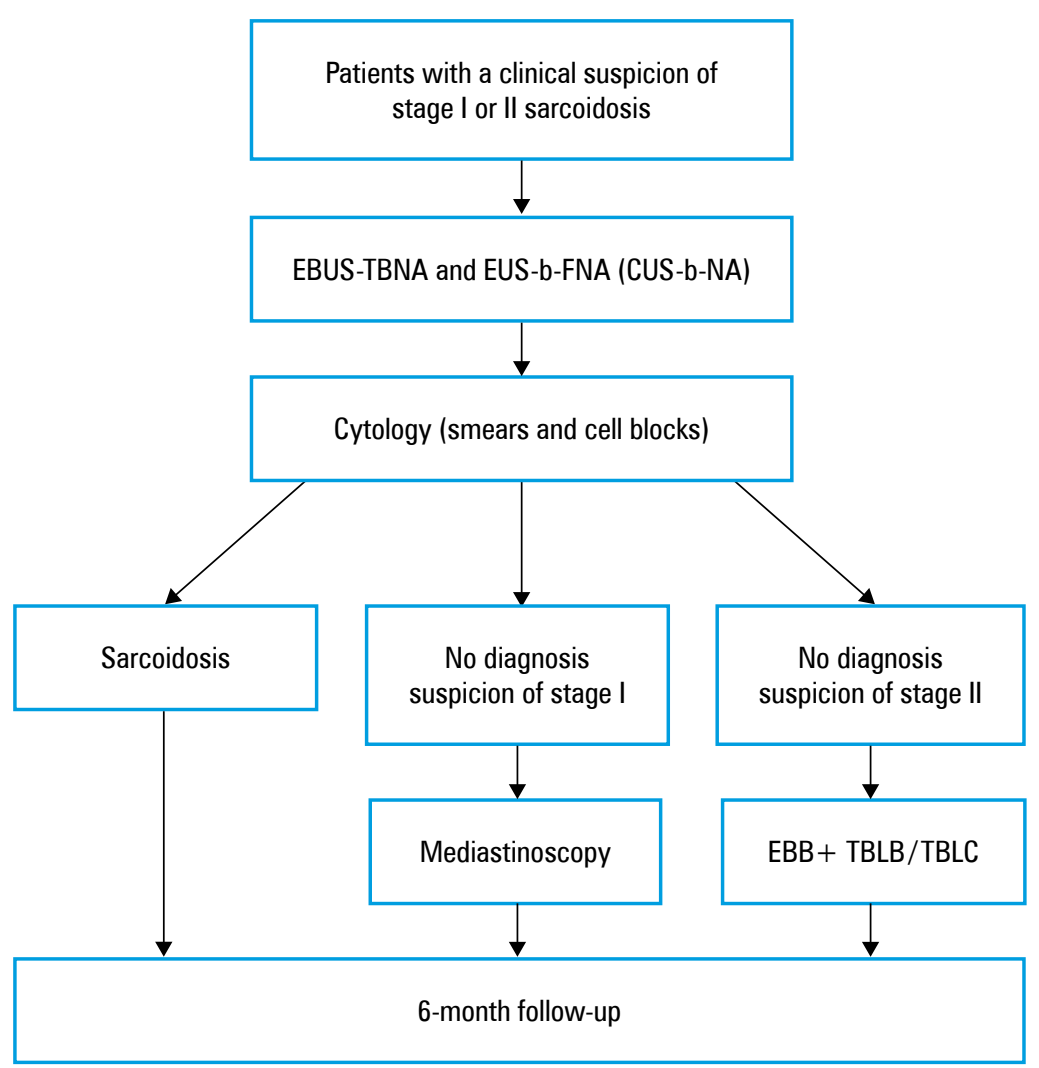

TABLE 1 Patient characteristics

\begin{tabular}{lll} 
Variable & & Value \\
Age, y, mean (SD) & & $42(8.5)$ \\
\hline Sex & Male & $33(66)$ \\
\cline { 2 - 3 } & Female & $17(34)$ \\
\hline Short diameter of the lymph node, mm, mean (SD) & & $15.2(6.2)$ \\
\hline Sarcoidosis & Stage I & $25(50)$ \\
\cline { 2 - 3 } & Stage II & $25(50)$ \\
\hline
\end{tabular}

Data are presented as number (percentage) unless otherwise indicated.

lesions, mostly micronodules on CT scans in the upper and middle lobes, typical of sarcoidosis.

Patient characteristics are presented in TABLE 1. In all patients, CUS-b-NA was performed in 204 mediastinal and hilar nodal stations in total, with EBUS-TBNA as the first procedure. There were 90 mediastinal (station 7, 50; station 4R, 24; station $4 \mathrm{~L}, 14$; station $2 \mathrm{R}, 2$ ) and 13 hilar (station 10R, 4; station $11 \mathrm{R}, 3$; station $11 \mathrm{~L}, 6$ ) nodal stations biopsied by EBUS-TBNA. There were also 101 mediastinal stations (station 7, 50; station 4R, 8; station 4L, 38; station 8, 3; station 2R, 1; station 2L, 1) biopsied by EUS-b-FNA. The cytological material was prepared for smears and CBs in all cases. Granuloma detection rate was as follows: EBUS-TBNA, 72\% (36 out of 50 patients), EUS-b-NA, $66 \%$ (33 out of 50 patients), and CUS-b-NA, $86 \%$ (43 out of 50 patients). In 7 patients (14\%), specimens were nondiagnostic due to a low number of cells. In 1 patient (2\%) with stage I sarcoidosis, a complete regression of adenopathy was observed at 6-month follow-up and MS was not performed. In the next 6 patients (12\%) with stage II sarcoidosis, conventional bronchoscopic diagnostic workup was performed including EBB and TBLB. In 4 patients (8\%), granulomas were detected by TBLB, and in 2 patients (4\%), a partial regression and stable clinical outcome of parenchymal micronodules and adenopathy at 6-month follow-up were reported, so TBLC was not performed in those patients. In all patients, tuberculosis cultures taken from all endoscopy bioptic methods were negative. A clinical and radiological assessment at follow-up did not show any signs of lymphoma, lung cancer, or other diseases presenting granuloma reactions in lymph nodes. A total of 15 patients (30\%) with stage I sarcoidosis had a spontaneous remission of adenopathy. 
TABLE 2 Calculated sensitivity, accuracy, negative predictive value, and granuloma detection rate per patient of endosonographic techniques in the diagnostic workup of stage I and II sarcoidosis

\begin{tabular}{lllll} 
Endosonographic technique & Sensitivity & Accuracy & NPV & GDR, \% \\
EBUS-TBNA (S+CB) & $76.6(61.97-87.70)$ & $78(64.04-88.47)$ & $21.43(13.99-31.39)$ & 72 \\
\hline EBUS-TBNA (S) & $55.32(40.12-69.83)$ & $58(43.21-71.81)$ & $12.5(9.41-16.41)$ & 52 \\
\hline EUS-b-FNA (S+CB) & $70.21(55.11-82.66)$ & $72(57.51-83.77)$ & $17.65(12.14-24.95)$ & 66 \\
\hline EUS-b-FNA (S) & $53.19(38.08-67.89)$ & $56(41.25-70.01)$ & $12(9.14-15.61)$ & 50 \\
\hline CUS-b-NA (S+CB) & $91.67(80.02-97.68)$ & $92,16(81.12-97.82)$ & $42.86(22.69-65.71)$ & 86 \\
\hline CUS-b-NA (S) & $76.6(61.97-87.70)$ & $78(64.04-88.47)$ & $21.43(13.99-31.39)$ & 72 \\
\hline CUS-b-NA (CB) & $68.09(52.88-80.91)$ & $70(55.39-82.14)$ & $16.67(11.64-23.29)$ & 64 \\
\hline CUS-b-NA (S+CB) stage I & $100(85.75-100)$ & $100(86.28-100)$ & 100 & 96 \\
\hline CUS-b-NA (S+CB) stage II & $82.61(61.22-95.05)$ & $84(63.92-95.46)$ & $33.33(17.02-54.92)$ & 76 \\
\hline
\end{tabular}

Data are presented as percentage $(95 \% \mathrm{Cl})$ unless otherwise indicated.

Abbreviations: CB, cell block; GDR, granuloma detection rate; NPV, negative predictive value; S, smear; others, see FIGURE 4

A partial regression of diffuse parenchymal lesions in 7 patients $(14 \%)$ with stage II sarcoidosis was noted. At 6-month follow-up, all patients were in a stable clinical condition.

Calculated sensitivity, accuracy, negative predictive value, and granuloma detection rate (GDR) per patient are presented in TABLE 2. Specificity and positive predictive value were $100 \%$ for all methods.

Sensitivity, accuracy, and GDR of EBUS-TBNA and EUS-b-FNA are comparable ( $P=0.52$, $P=0.47, P=0.63$, respectively) whereas those of CUS-b-NA were higher than those of EBUS-TBNA $(P=0.005, P=0.004, P=0.016$, respectively) and of EUS-b-FNA ( $P=0.001, P=0.001$, $P=0.002$, respectively).

Sensitivity, accuracy of CUS-b-NA was higher in stage I than in stage II sarcoidosis $(P=0.04$, $P=0.04$, respectively).

Sensitivity, accuracy, and GDR of cytological smears and the $C B$ technique were comparable ( $P=0.36, P=0.33, P=0.48$, respectively). However, after comparing sensitivity, accuracy, and GDR of the combination of smears and $C B$ to smears alone, the differences were significant for all biopsy methods: EBUS-TBNA $(P=0.002$, $P=0.001, P=0.002$, respectively), EUS-b-FNA ( $P=0.003, P=0.004, P=0.008$, respectively), and CUS-b-NA ( $P=0.008, P=0.006, P=0.016$, respectively).

Sensitivity, accuracy, and GDR of CUS-b-NA calculated per station were higher when the subcarinal lymph nodes were biopsied in comparison with all other lymph node stations $(P=0.001$, $P=0.001, P=0.0001$, respectively).

No procedure-related complications were noted during CUS-b-NA biopsies.

DISCUSSION Cytological confirmation is of great importance in the diagnostic workup of sarcoidosis. Endosonography-guided biopsy techniques have higher efficacy than conventional methods (EBB and TBLB). ${ }^{6,7,11,16}$ The preferred method of obtaining cytological material is EBUS-TBNA. ${ }^{17,18}$ Transesophageal approach seems to be underestimated and used less often. Literature search showed no advantage of any of these methods: both sensitivity and specificity of EBUS-TBNA and EUS-b-FNA are comparable. ${ }^{7,10,11}$ Choosing EBUS-TBNA over EUS-b-FNA might be related with the preferences of pulmonologists diagnosing sarcoidosis, as they are more acquainted with the endobronchial rather than endoesophageal approach.

In the current study, sensitivity and accuracy $76.6 \%$ and $70 \%$ for EBUS-TBNA, $78 \%$ and $72 \%$ for EUS-b-FNA, respectively, did not differ from those presented in the literature, ${ }^{6-11,19}$ and lack of differences between sensitivity of EBUS-TBNA and EUS-b-FNA was confirmed $(P=0.52)$.

A slightly lower than expected sensitivity of EUS-b-FNA might be connected with group characteristics. A relatively high percentage (50\%) of enrolled patients had stage II sarcoidosis and only in this subgroup, false-negative results were noted. A lower sensitivity and accuracy of CUS-b-NA in stage II subgroup influenced the result of the entire group. The lower yield of CUS-b-FNA in detecting granulomas in the smears might be caused by the fact that CBs were prepared first and smears were prepared second with the remaining material. The mean (SD) diameter of visualized lymph nodes was $15.2 \mathrm{~mm}(6.2 \mathrm{~mm})$ and quite often normal-sized lymph nodes were fibrotic and hard to puncture. Better efficacy of EBUS-TBNA over EUS-b-FNA might be explained by the bias related to the study design. Endoscopy procedures were performed mostly by pulmonologists and EBUS-TBNA was preferred as the first procedure. Considering the fact that one needle was used for the whole CUS-b-NA procedure and the first few biopsies were taken transbronchially, the material obtained by EUS-b-FNA might be of slightly worse quality.

Our study showed high sensitivity (91.7\%) and accuracy (92.2\%) of CUS-b-NA. Diagnostic yield of this method was higher than that of EBUS-TBNA and EUS-b-FNA alone $(P=0.005$, $P=0.001$, respectively). Does it mean that CUS-b-NA should be considered as the standard in 
cytological diagnostic workup of mediastinal lymphadenopathy? As this advantage of CUS-b-NA may be the effect of an increased number of biopsies and summary of the results, further studies are needed.

The combined endosonographic approach seems to be a safe biopsy method. No complications were noted in our group. However, considering potentially increased complication risk connected with higher number of biopsies, in our opinion, CUS-b-NA should be reserved for patients with stage II sarcoidosis, especially when lymph nodes are relatively small on computed tomography and obtaining biopsy material might be more difficult.

There are not many papers ${ }^{4,7}$ on the influence of the tissue material preparation on the diagnostic yield of the biopsy method. In most cases, cytological material obtained by needle biopsy is used to prepare smears. In our study, we compared diagnostic yields of these 2 cytological methods (smears and $\mathrm{CB}$ ) and we did not find differences $(P=0.36)$. However, we managed to show an increase of the diagnostic yield when these 2 techniques were joined regardless of the biopsy method $(P=0.008)$. Most of studies did not show clearly which cytological method, smear or $C B$, was used. 6,7,14,16 Our data shows that $C B$ preparation should be considered as the recommendation in the diagnostic workup of sarcoidosis.

The other clinical question we tried to answer in this trial was whether there is an association between the lymph node localization (station) and diagnostic yield of the endosonographic biopsy. Our results indicate an important role of the subcarinal lymph node station (7) biopsy in obtaining cytological diagnosis of sarcoidosis. The diagnostic yield of CUS-b-NA would have been lower if lymph nodes of station 7 had not been biopsied $(P=0.001)$. Perhaps biopsy of these lymph nodes should be recommended as mandatory. In current practice, biopsies of subcarinal lymph nodes are performed most frequently either transbronchially or transesophageally probably due to its reachability.

Study limitations A relatively small number of patients in whom final analysis was performed is one of the disadvantages of our study. The results presented in our paper should be considered for further meta-analyses and investigations in larger groups of patients. It should be emphasized that the guidelines for invasive procedures are rarely based on trials with a large number of patients. More often, meta-analyses of many small trials are made.

The GRANULOMA global study, ${ }^{6}$ the largest study on this topic including 354 patients (155 in the endosonography arm), was performed in 14 centers and 8 countries.

A very important paper by Oki et $\mathrm{al}^{11}$ which implemented and stated a new EUS-b method for the diagnostic workup of stage I and II sarcoidosis was based on the analysis of 33 patients.
The other limitation of the study protocol was the use of the same needle for the whole CUS-b-FNA procedure. As the ability of obtaining the material decreases with the number of biopsies performed, it could influence the diagnostic yield of the procedure / processing method used as the second one.

Finally, there was lack of histological confirmation in 3 patients ( 1 with stage I and 2 with stage II sarcoidosis). The final diagnosis of sarcoidosis in these cases was based on clinical outcome, radiology exclusion of the other diseases with granulomatous inflammation, and the radiological remission at follow-up.

Conclusions Even though both EBUS-TBNA and EUS-b-FNA are efficient in stage I and II sarcoidosis, the diagnostic yield of CUS-b-NA is higher than that of any of those techniques used alone. The preparation of cytological material including CB technique and a choice of subcarinal lymph node station for biopsy increases diagnostic efficacy.

\section{ARTICLE INFORMATION}

CONTRIBUTION STATEMENT AF, MG, JS, DC, and AS conceived the concept of the study and contributed to the design of the research. AF, $M G, A S, J W, A J, J P$, and WZ were involved in data collection. AF, AS, MG, $J W$, and $A C$ analyzed the data. JP and WZ performed pathological assessment of all samples. AF, MG, AS, and JS edited the manuscript. All authors approved the final version of the manuscript.

\section{CONFLICT OF INTEREST None declared}

OPEN ACCESS This is an Open Access article distributed under the terms of the Creative Commons Attribution-NonCommercial-ShareAlike 4.0 International License (CC BY-NC-SA 4.0), allowing third parties to copy and redistribute the material in any medium or format and to remix, transform, and build upon the material, provided the original work is properly cited, distributed under the same license, and used for noncommercial purposes only. For commercial use, please contact the journal office at pamw@mp.pl.

HOW TO CITE Filarecka A, Gnass M, Wojtacha J, et al. Usefulness of combined endobronchial and endoscopic ultrasound-guided needle aspiration in the diagnosis of sarcoidosis: a prospective multicenter trial. Pol Arch Intern Med. 2020; 130: 582-588. doi:10.20452/pamw.15399

\section{REFERENCES}

1 Baughman RP, Culver DA, Judson MA. A concise review of pulmonary sarcoidosis. Am J Respir Crit Care Med. 2011; 183: 573-581. ¿

2 Bradley B, Branley HM, Egan JJ, et al; British Thoracic Society Interstitial Lung Disease Guideline Group, British Thoracic Society Standards of Care Committee; Thoracic Society of Australia; New Zealand Thoracic Society; Irish Thoracic Society. Interstitial lung disease guideline: the British Thoracic Society in collaboration with the Thoracic Society of Australia and New Zealand and the Irish Thoracic Society. Thorax. 2008; 63 (suppl. 5): 1-58.

3 Govender P, Berman JS. The diagnosis of sarcoidosis. Clin Chest Med. 2015; 36: 585-602. ¿

4 Chee A, Khalil M, Stather DR, et al. Cytologic assessment of endobronchial ultrasound-guided transbronchial needle aspirates in sarcoidosis. J Bronchology Interv Pulmonol. 2012; 19: 24-28.

5 Statement on sarcoidosis. Joint Statement of the American Thoracic Society (ATS), the European Respiratory Society (ERS) and the World Association of Sarcoidosis and Other Granulomatous Disorders (WASOG) adopted by the ATS Board of Directors and by the ERS Executive Committee, February 1999. Am J Respir Crit Care Med. 1999; 160: 736-755.

6 von Bartheld MB, Dekkers OM, Szlubowski A, et al. Endosonography vs conventional bronchoscopy for the diagnosis of sarcoidosis: the GRANULOMA randomized clinical trial. JAMA. 2013; 309: 2457-2464. [

7 Kocoń $\mathrm{P}$, Szlubowski A, Kużdżał J, et al. Endosonography-guided fine needle aspiration in the diagnosis of sarcoidosis: a randomized study. Pol Arch Intern Med. 2017; 127: 154-162. ¿

8 Bugalho A, Szlubowski A. Endobronchial ultrasound in sarcoidosis: time to rethink the diagnostic strategy? Rev Port Pneumol. 2014; 20: 235-236.

9 von Bartheld MB, Veseliç-Charvat M, Rabe KF, Anema JT. Endoscopic ultrasound-guided fine-needle aspiration for the diagnosis of sarcoidosis. Endoscopy. 2010; 42: 213-217. 
10 Gnass M, Szlubowski A, Soja J, et al. Comparison of conventional transbronchial vs endobronchial, endoscopic ultrasound-guided needle aspiration in the diagnosis of pulmonary sarcoidosis: a randomized trial. Pol Arch Intern Med. 2015; 125: 321-328.

11 Oki M, Saka H, Kitagawa C, et al. Transesophageal bronchoscopic ultrasound-guided fine needle aspiration for diagnosis of sarcoidosis. Respiration. 2013; 85: 137-143.

12 Vilmann $\mathrm{P}$, Clementsen PF, Colella S, et al. Combined endobronchial and oesophageal endosonography for the diagnosis and staging of lung cancer. European Society of Gastrointestinal Endoscopy (ESGE) guideline, in cooperation with the European Respiratory Society (ERS) and the European Society of Thoracic Surgeons (ESTS). Eur Respir J. 2015; 46: 40-60.

13 Gnass M, Sola J, Filarecka A, et al. Initial Polish experience of flexible 19 gauge endobronchial ultrasound-guided transbronchial needle aspiration. Adv Respir Med. 2017; 85: 64-68.

14 Mooij-Kalverda KA, Crombag L, Szlubowski A, et al. EBUS vs EUS-B for diagnosing sarcoidosis: the International Sarcoidosis Assessment (ISA) RCT. Eur Resp J. 2018; 52 (suppl. 62): PA396.

15 Gnass M, Filarecka A, Pankowski J, et al. Transbronchial lung cryobiopsy guided by endobronchial ultrasound radial miniprobe in interstitial lung diseases: preliminary results of a prospective study. Pol Arch Intern Med. 2018; 128: 259-262. ᄃ

16 Pedro C, Melo N, Novais E Bastos H, et al. Role of bronchoscopic techniques in the diagnosis of thoracic sarcoidosis. J Clin Med. 2019; 8 : 1327.

17 Agarwal R, Srinivasan A, Aggarwal AN, Gupta D. Efficacy and safety of convex probe EBUS-TBNA in sarcoidosis: a systematic review and meta-analysis. Respir Care. 2012; 106: 883-892. $\pi$

18 Benzaquen S, Aragaki-Nakahodo AA. Bronchoscopic modalities to diagnose sarcoidosis. Curr Opin Pulm Med. 2017; 23: 433-438. ¿

19 Tremblay A, Stather DR, Maceachern P, et al. A randomized controlled trial of standard vs endobronchial ultrasonography-guided transbronchial needle aspiration in patients with suspected sarcoidosis. Chest. 2009; 136: 340-346. 